\title{
Optimisation and personalization technologies and algorithms for future transportation systems
}

\author{
Evangelos D. Bekiaris
}

The Transport sector is presently undergoing a rapid and fundamental change that affects all transportation modes and market segments. The main driving forces behind it rest at the confluence of ITS and Industry 4.0 related technologies, whereas new mobility schemes are emerging; such as Mobility-as-a-Service (MaaS) as well as Connected and Automated Vehicles (CAVs). Together they reflect a paradigm shift from vehicle ownership to vehicle use, as well as multimodal unmanned vehicle fleets. Predominant in this emerging mobility world is the concept of modular and adaptable services; that bridge the traveler mobility demand to the mobility services offer in a dynamic manner. Such a matching/ mapping though requires robust optimization algorithms and personalization techniques.

This Topical Collection contains 4 papers that together cover key aspects of applying optimization and personalization to different parts of the mobility system. The papers stem from a selection of papers from ICTR2017 Conference (International Conference on Transportation and Research), that took place on 28-29 September 2017 in Thessaloniki, Greece [1].

The first paper performs a benchmarking in the area of ITS optimization for Public Transport (PT) operations. In the context of the Eurobarometer Survey (2014), Commissioner Violeta Bulc said: "Today's survey shows that good infrastructure, better connections, and cheaper tickets are the main concerns of EU citizens. That is why we need to remove technical and administrative barriers to ensure that transport services can really operate across the whole $E U$, without national boundaries. Also we cannot assume that transport services will always be there, or be safe, unless we maintain them. Transport is about people. That is why in all of my initiatives, the main objective will be to contribute to

Correspondence: abek@certh.gr

Centre for Research and Technology Hellas, Hellenic Institute of Transport, Thessaloniki, Greece

c) The Author(s). 2019 Open Access This article is distributed under the terms of the Creative Commons Attribution 4.0 International License (http://creativecommons.org/licenses/by/4.0/), which permits unrestricted use, distribution, and reproduction in any medium, provided you give appropriate credit to the original author(s) and the source, provide a link to the Creative Commons license, and indicate if changes were made. travellers needs and to set the conditions for the European transport economy to flourish."The survey also revealed that convenience is by far the main reason for choosing a specific means of transportation for everyday and long journeys (both $61 \%$ ), followed by speed (respectively $31 \%$ and $41 \%)$ and price (12\% and18\%). ITS is a prominent tool to achieve the above societal goal. And it is even pivotal for the emerging PT fleets automation.

The second paper focuses upon optimizing the safety and performance of private cars by designing and personalizing the functionality of Advanced Driver Assistance Systems (ADAS). According to most recent reports [2], in 2014, the largest penetration rate of ADAS in the market was still in Europe, with a penetration rate of around $9.86 \%$. Thus, although Cooperative Intelligent Transportation Systems (C-ITS) may have a great impact on road safety, their extremely small penetration practically nullifies their impact. One of the main reasons behind this fact is related to the usability of these systems; with many drivers reporting too early or too many warnings whereas others claiming too late response. Clearly, the "one size fits all" concept of today's ADAS does not suffice to serve the very varying driving cohorts needs; from the sporty driving style of an experienced commuter to the defensive one of an elderly driver in an unfamiliar environment.

The third paper introduces optimization in Traffic Management Schemes. In today's large-scale urban networks major dynamic changes are occurring, involving travel demand, model choice motivations and share, route choices (affected also by navigation and routing systems) and traffic flow evolutions. As novel demand prediction models need to be able to deal with "uncertainty", also urban TMC needs to be traffic responsive and apply traffic simulation optimization effectively into real city traffic networks in real time. Such modeling goes along with much more complete solutions to traffic control, able to handle all the many new and old streams of data and how to best utilize them for 
traffic management, influencing also the traffic demand side.

The fourth paper concerns driving behavior modelling for car following in mixed traffic; especially in the case of weak lane-discipline conditions. Although this is based on the traffic conditions and behaviour of developing countries, it might be a very relevant concept for the mixed traffic conditions and interactions between human-driven and partly or fully automated vehicles in the future. Especially in its transition phase, the co-existence of all road users, the motorized and non-motorized as well as the automated and the non-automated, requires optimized and even personalized traffic behavior modelling both for longitudinal and lateral road movements.

The four papers in this Topical Collection together touch upon different aspects of the same major challenge; how to optimally employ optimization and personalization principles to enable the safe and smooth rapid transition to the transport world of tomorrow; a totally new era, we propose to call "Transport 4.0"; as a direct counterpart element of the emerging Industry 4.0 notion. Both concepts are characterized by automation, flexibility, recyclability and the substitution of products by services.

\section{ITS use for public transport operations optimization}

Christina Iliopoulou and Konstantinos Kepaptsoglou perform a state of the Art on Intelligent Transportation Systems (ITS) implementation in Public Transport (PT); with emphasis on Automatic Vehicle Location (AVL) and Automatic Passenger Count (APC) systems; focusing on both planning and operational tasks. They analyze the relevant ITS impacts to the strategic, tactical and operational levels of PT operations. Some of the specific related tasks that are analyzed for the impact of ITS include transit assignments, network design, optimal timetabling, origin-destination and transfer interfaces, activity modeling and vehicles scheduling. Furthermore, the impact of ITS technologies and algorithms to real-time PT operations is explored, from real time trip planning to real-time control.

The paper recognizes major research gaps and challenges in the use of ITS for PT operations optimization, including:

- Additional data processing required,

- Lack of integration among various data sources,

- Different degrees of ITS penetration into vehicle fleets,

- Lack of transfer of optimization applications to real fleet operation,

- Increased computational requirements,

- Limiting operators' data sharing policies.
Based on the above, the authors propose specific research opportunities (at strategic, tactical and operational level; as well as for real-time operations) but also describe the inherent research limitations due to data quality, lack of data supplementarity and required computational effort.

Last but not least, emerging trends in PT operations optimization are described, with emphasis on emerging data sources exploration and the emergence of integrated transit models.

\section{Personalization of ADAS warnings for acceptance, safety and systems penetration enhancement}

Maria Panou identifies the need to optimize and even personalize the warnings of ADAS in order to maximize driver acceptance, enhance road safety and facilitate their rapid deployment. Work focuses upon personalizing the algorithms of Collision Avoidance Systems (CAS); however the overall concept is easily transferable to other ADAS types. Work starts with the identification of the major parameters that influence the CAS performance and their clustering to those that can be adapted/ personalized in a static (based upon driver residual abilities and preferences), a semi-dynamic (based upon context of use) and a dynamic (based upon evolving driving style and conditions) manner. For all of them, a minimum safety threshold has to be set; in order to guarantee the safe operation of CAS.

Based on these parameters, three overall personalized functions are developed, corresponding to time to collision, time headway and reaction time personalization algorithms. Relevant algorithms for those functions are being developed and applied in an on-road test experiment with 10 drivers; using an equipped research car. The personalized parameters for each driver were calculated under normal real driving conditions as well as through emergency braking sessions. Then, the "standard" CAS warnings were compared to the personalized ones based upon different personalization principles (i.e. CAS triggered by personalized time to collision, time headway or reaction time respectively). Test results showed that personalized warnings were more accepted in general by the drivers, whereas individual preferences upon best personalization criteria differed. In none of the tested personalized systems were safety conditions violated. And the more the driver's driving style varied from the "standard" condition (i.e. driving closer to the lead vehicle or leaving bigger distance from it than the "average" supposed by the CAS developer); the more he or she appreciated the system. In spite of varying system triggering distances and times, overall drivers' trust to system performance remained high. This proves the vast opportunity of resulting in more effective and even affective and persuasive ADAS through personalization of their 
warning thresholds; thus reaching higher market penetration and use.

\section{Traffic management optimization through coordinated traffic-response signal control algorithms}

Diamantis Manolis, Christina Diakaki, Ioannis Papamichail and Markos Papageorgiou developed a simulation based novel Traffic-responsive Urban Control (TUC) strategy for higher efficiency in saturated large-scale networks. Several optimization methodologies have been employed, such as relocating unused green time to stages serving high demand or preserving TUCordered offsets or combination of the above features. Microscopic simulation was based upon the AIMSUN simulation software, utilizing the traffic data of a real city network; that of Chania in Greece. Three different demand scenarios were run, from light traffic conditions to dense and, ultimately, congested ones. Each one was run three times, with different TUC configurations; based upon different algorithms. The one based upon the relocation of unused green time proved to be the most efficient approach. Still, testing under real city conditions is required for the full and in-depth assessment of the proposed algorithms.

\section{Mixed traffic modeling optimization under weak lane-discipline conditions}

Vasileia Papathanasopoulou and Constantinos Antoniou performed driver behavior modeling in mixed traffic with weak lane-discipline trajectory data; using as reference the Gipps model on car following behavior. Both longitudinal and lateral movements are simulated and modeled. Such flows are typical of developing countries and traffic data from India have been used to build and assess the relevant model. However, they are also relevant to roads with non-optimal lane markers (typical in South Europe among other areas) or even to future mixed flows of automated and non-automated cars; where non automated/non C-ITS equipped cars may be mimicking the behavior of the automated ones. The data-driven model developed draws temporary virtual lanes and identifies dynamically leader-follower pairs, allowing also for multiple leaders for the same follower. The developed model results in better speed prediction than the reference Gipps model. As future prospects to further enhance the accuracy and prediction validity of the model, employment of swarm-like and crowd simulation models is planned. One of the major benefits of the proposed model concerns the ability to predict traffic with high heterogeneity of driver behavior (i.e. in touristic areas) and vehicle types (i.e. in mixed vehicle automation flows).

\section{Conclusions}

The range of topics within this Topical Collection demonstrates the range of approaches that can be employed to move from the saturated and unsafe urban traffic environments of today to the new mobility era of tomorrow. Instead of taking a futuristic approach and looking at the optimization of not yet existing traffic environments (i.e. automated vehicles and traffic management of automated vehicle flows), this TC takes a more pragmatic and evolution based approach. It benchmarks and develops strategies, algorithms and models for the optimal uptake of the most mature recent ITS technologies, in order to move from today's level 0 of automation to level 1 (with ADAS) and 2 (with automation in specific functions only) traffic environments, with connected cars and public transport under efficient traffic planning, operation and management regimes. Such algorithms and systems open us the door towards the future of urban traffic. According to an OECD/ITF report [3], up to 9 out of 10 conventional cars could become redundant under certain circumstances. This will lead to freeing public space, by increasing urban mobility depending on the choice of vehicle type, the level of penetration and the availability of high-capacity PT to complement the shared self-driving car fleet. UITP Policy Brief [4] highlights that there are various applications for autonomous vehicles as part of a diversified PT system, which will enable performing all demanded trips with $80 \%$ fewer cars. But drivers and travelers alike need to be prepared for such a revolution. The EC 2015 Eurobarometer survey [5] showed that $61 \%$ of participants throughout the EU expressed not feeling comfortable travelling with driverless cars, while they were more positive to the option of transporting goods using such vehicles, while a recent relevant survey in the US [6] found that $64 \%$ of respondents expressed concern about sharing the road with driverless cars. However, acceptance of automation in the driving task seems to be evolving with time as, according to the 2017 [7] and 2018 [8] Deloitte global automotive consumer studies, people throughout the world are becoming convinced that travelling with autonomous vehicles is safe, with the acceptance rate going from $45 \%$ to $72 \%$ in Germany and from $37 \%$ to $65 \%$ in France (in just 1 year!). Optimizing/personalizing the current vehicles and traffic flows operation by the best use of ITS, as approached within this TC, constitutes the most secure and straightforward way to enhance public awareness and acceptance on relevant technologies and prepare for the future.

\footnotetext{
Publisher's note

Springer Nature remains neutral with regard to jurisdictional claims in published maps and institutional affiliations.
}

Author's contributions

The author read and approved the final manuscript. 


\section{Competing interests}

The author declares that he has no competing interests.

Received: 9 July 2019 Accepted: 9 July 2019

Published online: 31 July 2019

\section{References}

1. [Online]. Available: https://www.ictr.gr/Documents2017/ICTR2017_ Programme_web.pdf. Accessed 30 Nov 2017.

2. Chisult Insight. (2015). Research on global \& China advanced driver assist systems (ADAS) industry. RnR Market Research.

3. Martinez, L., \& Crist, P. (2015). Urban Mobility System Upgrade-How shared self-driving cars could change city traffic. International Transport Forum, Paris.

4. UITP (2017). Policy Brief: Autonomous vehicles: a potential game changer for urban mobility. Retrieved from: https://www.uitp.org/sites/default/files/cckfocus-papers-files/PolicyBrief_Autonomous_Vehicles_LQ_20160116.pdf.

5. Eurobarometer, S. 427, Autonomous Systems. (2015). Conducted by TNS Opinion and Social at the request of the Directorate-General for Communications Networks, Content and Technology (DG CONNECT).

6. Advocates for highway and auto safety (2018). CARAVAN Public Opinion Poll: Driverless Cars. Retrieved from: https://saferoads.org/wp-content/uploads/2 018/01/AV-Poll-Report-January-2018-FINAL.pdf.

7. Deloitte global automotive consumer study (2017). Retrieved from: https:// www2.deloitte.com/de/de/pages/consumer-industrialproducts/articles/ global-automotive-consumer-study.html.

8. Deloitte global automotive consumer study (2018). Retrieved from: https:// www2.deloitte.com/it/it/pages/consumer-business/articles/cipautomotivetrends-millennials-consumer-study.html

\section{Submit your manuscript to a SpringerOpen ${ }^{\odot}$ journal and benefit from:}

- Convenient online submission

- Rigorous peer review

- Open access: articles freely available online

High visibility within the field

- Retaining the copyright to your article 